\title{
Structural Ordering of Molybdenum Disulfide studied via Reactive Molecular Dynamics
}

\section{Simulations}

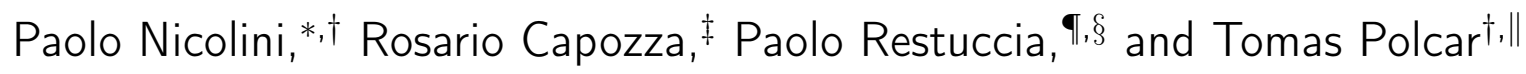 \\ $\dagger$ Department of Control Engineering, Faculty of Electrical Engineering, Czech Technical \\ University in Prague, Karlovo náměstí 13, 121 35, Prague 2, Czech Republic \\ $\ddagger$ Istituto Italiano di Tecnologia-IIT, Via Morego 30, 16163 Genova, Italy \\ IDipartimento di Scienze Fisiche, Informatiche e Matematiche, Università di Modena e \\ Reggio Emilia, via Campi 213/A, 41125 Modena, Italy \\ $\S C N R$-Institute of Nanoscience, S3 Center, via Campi 213/A, 41125 Modena, Italy \\ $\| n C A T S$, Faculty of Engineering and Environment, University of Southampton, \\ Southampton SO17 1BJ, United Kingdom \\ E-mail: nicolpao@fel.cvut.cz
}

Phone: +42022435 7442. Fax: +420224918646

\begin{abstract}
Molybdenum disulfide is a well known and effective lubricant, providing extremely low values of the coefficient of friction. It is known that the sliding process may induce structural transformations of amorphous or disordered $\mathrm{MoS}_{2}$ to the crystalline phase with basal planes oriented parallel to the sliding direction, which is optimal for reducing friction. However, the key reaction parameters and conditions promoting this structural transformation are still largely unknown. We investigate, by employing reactive molecular dynamics simulations, the formation of $\mathrm{MoS}_{2}$ layers from an amorphous phase as
\end{abstract}


a function of temperature, initial sample density and sliding velocity. We show that the formation of crystalline ordered structures can be explained in the framework of classical nucleation theory as it predicts the conditions for their nucleation and growth. These results may have important implications in the fields of coating and thin film deposition, tribology, and in all technological applications where a fast and effective structural transition to an ordered phase is needed.

\section{Keywords}

molybdenum disulfide, ordering process, crystal formation, molecular dynamics simulations, classical nucleation theory

\section{Introduction}

Molybdenum disulfide $\left(\mathrm{MoS}_{2}\right)$ is a material that has been known since ancient times. ${ }^{1}$ It occurs in nature as the mineral molybdenite. It has a characteristic layered structure and, within the individual lamellae, the repetitive unit is a triangular bipyramid with one molybdenum at the center and six sulfur atoms on the vertices. ${ }^{2}$ Strong covalent bonds act within the layers, whilst lamellae are held together with weak van der Waals forces only.

The chemical and physical properties of molybdenum disulfide have been exploited in many different fields. $\mathrm{MoS}_{2}$ has found wide application in catalysis (e.g., for the hydrogen evolution, ${ }^{3}$ the hydrodesulfurization, ${ }^{4}$ the $\mathrm{CO}$ hydrogenation ${ }^{5}$ and the photochemical water splitting ${ }^{6}$ reactions), as a sensor (for gases, ${ }^{7}$ proteins and $\mathrm{pH}^{8}$ ), in the biomedicine area (for example as an antibacterial agent ${ }^{9}$ and as a near-infrared photothermal-triggered drug carrier for cancer therapy ${ }^{10}$ ), for the fabrication of transistor devices ${ }^{11}$ and photovoltaic cells, ${ }^{12}$ and finally as a solid lubricant under vacuum. ${ }^{13}$ For each application, a particular structure is needed, including single-to-few layer architectures, nano-objects (e.g., fullerene-like or

nanotube particles) and materials exposing a large number of edge sites. In this regard, 
different fabrication techniques have been developed. ${ }^{14}$ Broadly speaking, for electronics, sensing and catalysis applications, wet-chemistry or chemical vapor deposition techniques are more often employed since they allow us to produce materials with tailored structural properties. On the other hand, in the tribological field, fabrication via magnetron sputtering is preferred, even though it generally offers less control over the crystallinity of the produced coatings (in some cases it leads to the growth of mixed crystalline structures ${ }^{15-20}$ and in others to more amorphous materials $\left.{ }^{21-25}\right)$.

Molybdenum disulfide represents one of the best option for reducing friction. It has been used extensively for decades since it provides a friction coefficient in the order of $10^{-2}$ or lower, approaching the so-called superlubrication regime (i.e., a state of vanishing friction) under highly controlled conditions. ${ }^{18,26}$ The lubrication mechanism implies the formation of an "easy-shear" interface made of molybdenum disulfide basal planes oriented in the sliding direction. Such a situation is ubiquitous in $\mathrm{MoS}_{2}$-lubricated sliding contacts ${ }^{16,19,22-24,27-30}$ and can be achieved through two different processes: the reorientation of misaligned crystallites and the ex-novo formation of ordered structures from amorphous material. Furthermore, the process of layer formation has been observed for other compounds belonging to the family of transition metal dichalcogenides (TMDs, for example $\mathrm{WS}_{2}{ }^{31}$ and $\mathrm{WSe}_{2}{ }^{32}$ ), or in coatings where TMD compounds were co-sputtered with other metallic (e.g., $\operatorname{gold}^{33,34}$ or lead ${ }^{35}$ ) or non-metallic (typically carbon ${ }^{36}$ or nitrogen ${ }^{37}$ ) atoms or compounds. The exact mechanism of formation of the ordered TMD tribolayer is not yet known. Amorphous TMD materials can be transformed into their crystalline form by heating (annealing processes) ${ }^{25,38-42}$ or by irradiating the sample with laser or electron beams. ${ }^{43-46}$ Since dissipated frictional energy is mostly transformed into heat, it is tempting to attribute layer formation to a combinatorial effect of heating (crystallization) and shear (basal plane orientation).

In this paper, computational tools are used to unravel the formation of well-ordered crystalline layer during sliding. Computer simulations can help us progress toward this goal, considering that they have greatly contributed to the understanding of the structure, ${ }^{47,48}$ 
electronic properties, ${ }^{49}$ and reactivity ${ }^{50,51}$ of $\mathrm{MoS}_{2}$. However, to the best of our knowledge, only one study ${ }^{52}$ has addressed the question of how and when $\mathrm{MoS}_{2}$ undergoes an amorphous to layered transition as a consequence of sliding. We go beyond the rough approximations adopted in such a study by taking into account dispersive interactions between layers and the possibility of bond breaking. We performed non-equilibrium molecular dynamics simulations with a reactive many-body interaction potential, allowing us to observe the formation of $\mathrm{MoS}_{2}$ layers from amorphous structures. In addition, we systematically studied the effect of the reaction parameters (i.e., the system temperature, the imposed sliding speed and the density of the initial amorphous state) on the layer formation dynamics.

\section{Computational details}

All molecular dynamics (MD) simulations were carried out using the LAMMPS package ${ }^{53}$ and employing a simulation time step of 0.1 fs. Atomic interactions were calculated employing the AIREBO potential for $\mathrm{MoS}_{2} \cdot{ }^{54,55}$ This force field is reactive (i.e., it can properly model the occurrence of the formation and breaking of bonds) and it provides an accurate representation of the crystalline phases in terms of predicted structural and energetic properties. ${ }^{56}$ The atomic positions in the unit cell for the crystal phase were taken from diffraction experiments. ${ }^{2}$ The hexagonal cell was converted into a tetragonal one following the procedure described elsewhere ${ }^{57}$ and replicated 17 and 34 times in the $x$ and $y$ directions respectively. The structure was then optimized performing an energy minimization with the conjugate gradient method and allowing atomic positions as well as box size and shape to vary. The initial configurations were produced using the following procedure. Six $\mathrm{MoS}_{2}$ layers from the previous structure were taken and put in an equal number of simulation boxes for the melting process. The sizes of each box were the same as the optimized supercell in the $x$ and $y$ directions, whilst the value in the $z$ one was calculated in order to span the range of densities experimentally observed in sputtered coatings $\left(0.77-4.07 \mathrm{~g} \mathrm{~cm}^{-3}\right) .{ }^{23}$ The systems 
were then heated at $5300 \mathrm{~K}$ and at constant volume for $500 \mathrm{ps}$, controlling the temperature via a Nosé-Hoover ${ }^{58,59}$ chain of thermostats with a damping parameter of 10 fs and leading to the complete melting of the samples. After that, the systems were rapidly quenched to $300 \mathrm{~K}$ at constant volume in order to form amorphous structures. ${ }^{60}$ The samples were then equilibrated at room temperature and pressure applied in the $z$ direction $(50 \mathrm{GPa})^{1}$ through a Parrinello-Rahman barostat. ${ }^{61}$ The amorphous systems were then inserted between two rigid and crystalline layers (one atop and one underneath the disordered region), also adding $20 \AA$ of vacuum in the $z$ direction and minimizing the energy of the resultant configurations. The sandwiched systems were finally equilibrated for 3 ns at room temperature by imposing a normal load to the top layer (atomic positions of this layer in the $x$ and $y$ directions, as well as atomic positions of the bottom one in any direction, were kept fixed) until reaching a stabilized value of the system's total energy. The computational setup is depicted in Figure 1. The final configurations contain 10404 atoms and the simulation boxes have sizes of 93.1,

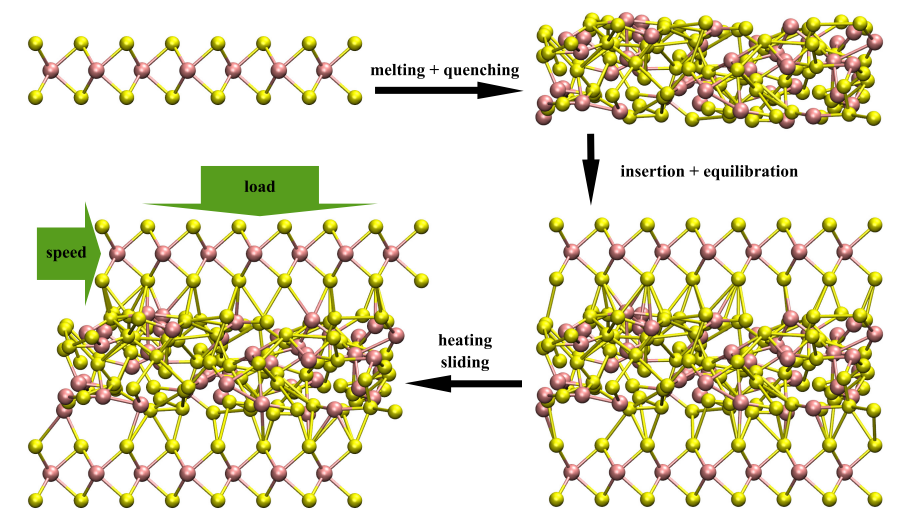

Figure 1: Schematic representation of the computational setup used in this study. Molybdenum and sulfur atoms are rendered in pink and yellow respectively. See the main text for more details.

107.5 and $38.4 \AA$ in the $x, y$ and $z$ directions respectively.

Non-equilibrium simulations were performed starting from these equilibrated configurations by keeping the atomic coordinates of the bottom layer fixed and by imposing a normal

\footnotetext{
${ }^{1}$ Preliminary calculations performed with 5 and $20 \mathrm{GPa}$ have revealed that the particular value chosen is necessary in order to observe the ordering transition within the time window achievable with MD simulations.
} 
load and sliding velocities in the $x$ direction to the atoms belonging to the rigid top layer (these atoms were able to move freely in the $y$ and $z$ directions). No constraints were applied to the central (amorphous) region. A Langevin thermostat ${ }^{62}$ with a damping parameter of $100 \mathrm{fs}^{2}$ was applied to these atoms by removing the motion of the center of mass from the computation of the temperature. In the first 20 ps of simulation the temperature was increased from $300 \mathrm{~K}$ to the target value and then kept constant for the subsequent 2 ns. We stress that the range of temperatures considered here is below the melting temperature for this force field under load and confinement conditions (the actual value of the melting temperature is $7600 \mathrm{~K}$; please see the Supporting Information - Section 1 for more details). Atomic positions, velocities and forces were stored every $0.5 \mathrm{ps}$ for subsequent data analysis. In order to follow the degree of crystallinity within the sample we used the atomic coordination number $(\mathrm{CN})$ as an order parameter. The definition is as follows ${ }^{63}$

$$
C N_{i}=\sum_{j}\left\{\exp \left[\kappa\left(d_{i j}-r_{c}\right)\right]+1\right\}^{-1}
$$

where $d_{i j}$ is the distance between atoms $i$ and $j, r_{c}$ and $\kappa$ are fixed parameters and the sum runs over atoms of different species (namely, if $i$ is a molybdenum atom, then $j$ indexes sulfur atoms and vice versa). Roughly speaking, the $\mathrm{CN}$ of an atom can be seen as the number of its nearest neighbors of different chemical nature. Parameters involved in the evaluation of the $\mathrm{CN}$, as well as other structural quantities (i.e., bond distances and bending angles) were calculated from equilibrium MD simulations (for more details about the derivation of such parameters and their explicit values, see the Supporting Information - Section 2). These data were used to implement a simple algorithm able to recognize the formation and growth of the crystal nuclei during the sliding dynamics. For more information about such an algorithm, please refer to the Supporting Information - Section 3.

\footnotetext{
${ }^{2}$ Different values of the damping parameter spanning four orders of magnitude were used in preliminary calculations showing negligible differences.
} 


\section{Results and discussion}

We started the study by performing a set of MD simulations at high load (50 GPa) and at different temperatures (i.e., 3000, 3200, .., 5800, $6000 \mathrm{~K}$ ) for the six material densities considered and without imposing a sliding speed. We decided to employ such high temperature and load values in order to speed up the simulations, thus being able to study the process within the time scale achievable with MD simulations. Time profiles of different physical quantities for a typical trajectory at $4000 \mathrm{~K}$ are reported in Fig. 2 (results from the other trajectories are available in the Supporting Information - Section 4). Looking at the panels
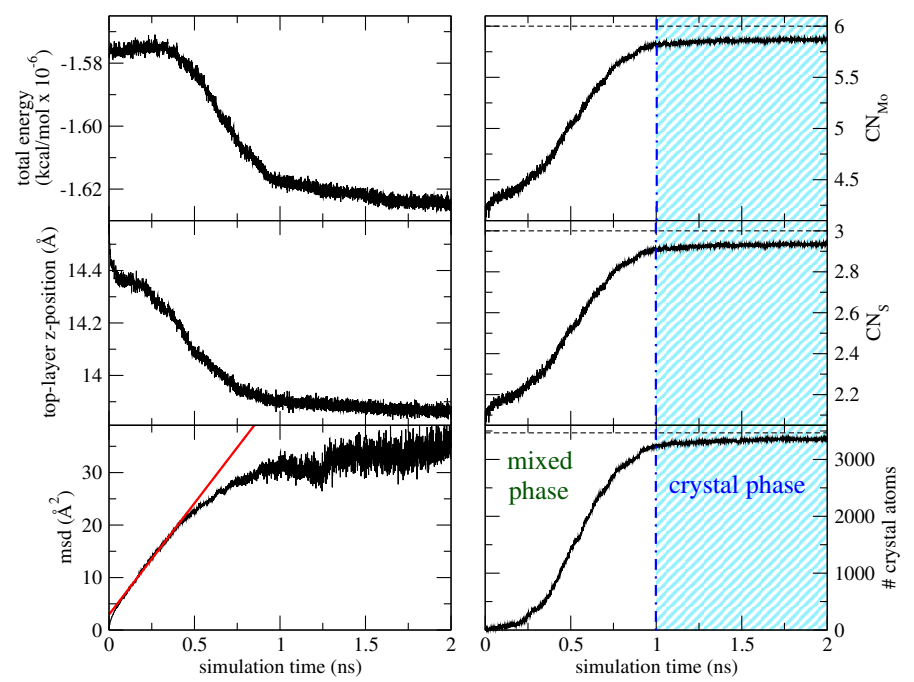

Figure 2: Time profiles of physical quantities calculated along a trajectory with load equal to $50 \mathrm{GPa}$, temperature equal to $4000 \mathrm{~K}$ and density equal to $2.09 \mathrm{~g} \mathrm{~cm}^{-3}$. Starting from the top right panel and moving in a clockwise direction, the panel show: average atomic coordination numbers for molybdenum and sulfur (respectively), number of crystalline atoms, mean square atomic displacement with respect to the initial position (the red line is the linear fit of the first part of the trajectory), position in the $z$ direction of the topmost rigid layer and total energy of the system, all reported as function of time. In the right panels, values corresponding to the crystal structure are plotted with dashed lines.

on the right, one can notice that from approximately 0.5 to 1 ns, both atomic coordination numbers $(\mathrm{CNs})$ and the number of atoms in the crystalline state monotonically increase until almost reaching limiting values. This basically indicates that the atoms in the amorphous region undergo a transition to a crystal phase. The transformation to the crystal phase follows a characteristic "s"-shaped profile characterized by a low rate of crystal formation 
both at the beginning and the end of transformation but a very rapid growth in between. Snapshots taken from the MD trajectory reported in Fig. 2 are shown in Fig. 3 (see the Supporting Information - Section 5 for more details). The initial slow rate is characterized
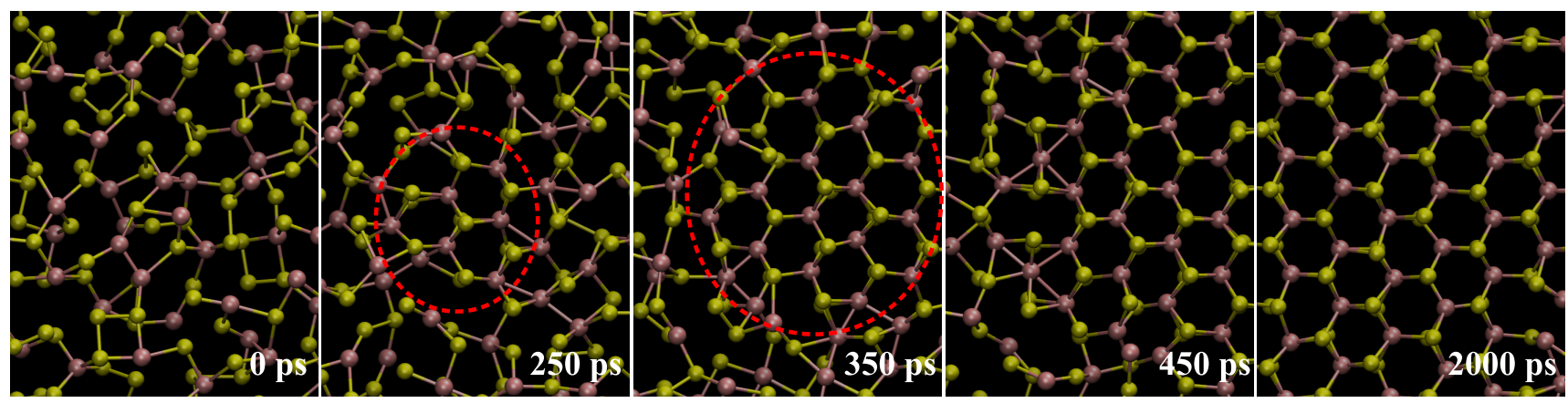

Figure 3: Top views of part of the initially amorphous region at various times. For the sake of clarity, atoms belonging to the rigid layers are not shown. Molybdenum and sulfur atoms are shown in pink and yellow respectively. Dashed red circles highlight the formation and growth of a crystal nucleus.

by the formation of a significant number of crystalline nuclei that expand with time (see Fig. 3). During the intermediate period the transformation is faster and at this phase the nuclei grow into large particles and consume the amorphous phase. The final phase, again with a slower rate, can be attributed to the small availability of untransformed material for further nucleation. The newly-formed large particles begin to touch one another, forming boundaries where growth stops. In addition, such a process can also be revealed by looking at the time profiles of the total energy of the system and the position in the $z$-direction of the topmost rigid layer (the transition from the amorphous to the crystal state leads to a decrease in the potential energy due to the formation of stronger chemical bonds, as well as an increase in the material density). In the left-bottommost panel of Fig. 2, the time profile of the mean square atomic displacement $(\mathrm{msd})$ is also reported. The msd values are calculated on the non-crystalline atoms only, in order to obtain the diffusion coefficient of $\mathrm{MoS}_{2}$ for the amorphous phase (red curve in the figure). After a very short initial transient interval, the trend is linear until the phase transformation takes place. After that, a behavior typical of a crystalline material is observed. 
As the graphs reported in the Supporting Information - Section 4 show, the scenario depicted above about the kinetics of crystal formation is qualitatively the same regardless of the values of the initial densities of the amorphous phase (only some differences in the initial and final times of the transition are observable with a non-monotonic trend). For this reason, in the subsequent analysis of the process kinetics, we may consider the data obtained with system at different initial coating densities as independent samples of an ensemble, therefore considering the average value associated to its uncertainty. On the other hand, the effect of the temperature is much more evident. At temperatures around $5000 \mathrm{~K}$, the process of layer formation takes place more quickly and the transition is complete after about 0.3 ns (the differences on the initiation time and duration of the ordering process among the samples with distinct initial density are also very small). At temperatures below $4500 \mathrm{~K}$, overall the process is slowed down, starting much later and proceeding with a slower rate. In some cases, the completion of the transformation is not observed in the nanosecond time scale explored in this study. At temperatures between 5000 and $6000 \mathrm{~K}$, the layer formation process is still very fast, but there is some delay in the initiation time, therefore making the overall time of transformation slower than at $5000 \mathrm{~K}$.

In order to rationalize these findings, we need to introduce the framework of the classical nucleation theory $(\mathrm{CNT}) .{ }^{64}$ Briefly, CNT describes the transition from a metastable phase to a more stable one passing through two related steps: firstly the formation of critical nuclei occurs (nucleation stage), and then such nuclei increase their size (growth stage). The nucleation process is governed by the interplay between the free energy difference between the two bulk phases (that represents the driving force of the phase transformation) and the surface energy (that the system needs to pay in order to create the interface). For example, for spherical nuclei, the former increases (in absolute value) with the cube of the particle radius, whilst the latter grows with the square of the particle radius. It is possible to demonstrate ${ }^{64}$ that there is a critical size $\left(r^{*}\right)$ for which particles bigger than such a value spontaneously evolve into the new phase, whilst nuclei smaller than $r^{*}$ shrink and dissolve 
again into the metastable phase. The free energy difference $\Delta G^{*}$ between a nucleus of radius $r^{*}$ and the metastable phase is the real barrier of the nucleation process, thus the nucleation rate $\dot{N}$ can be written as

$$
\dot{N}=A \exp \left(-\frac{\Delta G^{*}}{k_{B} T}\right)
$$

where $A$ is a pre-exponential factor (assumed to be independent of temperature), $k_{B}$ is the Boltzmann constant and $T$ is the temperature. The explicit expression for $\Delta G^{*}$ depends on the shape of the nuclei. Given the confinement in our setup, it is more natural to assume the formation of cylindrical nuclei instead of spherical ones, as done in isotropic environments. Therefore, the formula usually reported (see for example Ref. ${ }^{64}$ ) for the nucleation barrier (that is $\Delta G^{*}=\frac{16}{3} \pi \frac{\sigma^{3}}{\rho^{2} \Delta \mu^{2}}$, where $\sigma$ is the interface energy, $\rho$ the molar density and $\Delta \mu$ the difference in chemical potential between the two phases) becomes

$$
\Delta G^{*}=\frac{\pi h \sigma^{2}}{\rho \Delta \mu}
$$

where $h$ is the height of the cylindrical nuclei (the layer height, $6.145 \AA$ ). Unfortunately, the estimation of $\Delta G^{*}$ (and thus of $\sigma$ and $\Delta \mu$ ) is obtainable only through expensive and specific MD simulations, which are outside of the scope of this paper. Remaining on semiquantitative grounds, one can consider the first-order Taylor expansion of the free energy around the melting temperature. At constant pressure and composition, one can write $\Delta \mu \simeq-\frac{\Delta H_{m}}{T_{m}}\left(T-T_{m}\right)$, where $T_{m}$ represents the melting temperature and $\Delta H_{m}$ the molar melting enthalpy (at $T_{m}$ ). Both $T_{m}$ and $\Delta H_{m}$ can be estimated from the simulation results reported in the Supporting Information - Section 1. Eq. 2 then becomes ${ }^{3}$

$$
\dot{N} \simeq A \exp \left[-\frac{\pi h \sigma^{2} T_{m}}{\rho \Delta H_{m}\left(T_{m}-T\right) k_{B} T}\right]
$$

After nucleation, the growth of such nuclei takes place. This process is ruled by diffusion

\footnotetext{
${ }^{3}$ For a complete derivation of the formulas here reported, see the Supporting Information - Section 6.
} 
(at least for $T \ll T_{m}$ ) and the linear growth rate $\dot{G}$ takes the form ${ }^{64}$

$$
\dot{G}=\frac{D}{a}\left[1-\exp \left(-\frac{\Delta \mu}{k_{B} T}\right)\right] \simeq \frac{D}{a}\left\{1-\exp \left[-\frac{\Delta H_{m}\left(T_{m}-T\right)}{T_{m} k_{B} T}\right]\right\}
$$

where $D$ is the diffusion coefficient in the mestastable phase and $a$ is a characteristic length of the system (taken as the value of the lattice constant of the monolayer, $3.16 \AA$ ). The diffusion coefficient as a function of the temperature has been obtained from the fittings as shown in Fig. 2 (left-bottom panel; additional details about the calculation of the diffusion coefficient can be found in the Supporting Information - Section 7). The behaviour of $D$ and $\dot{G}$ as a function of temperature are both shown in Fig. 4. The growth rate increases

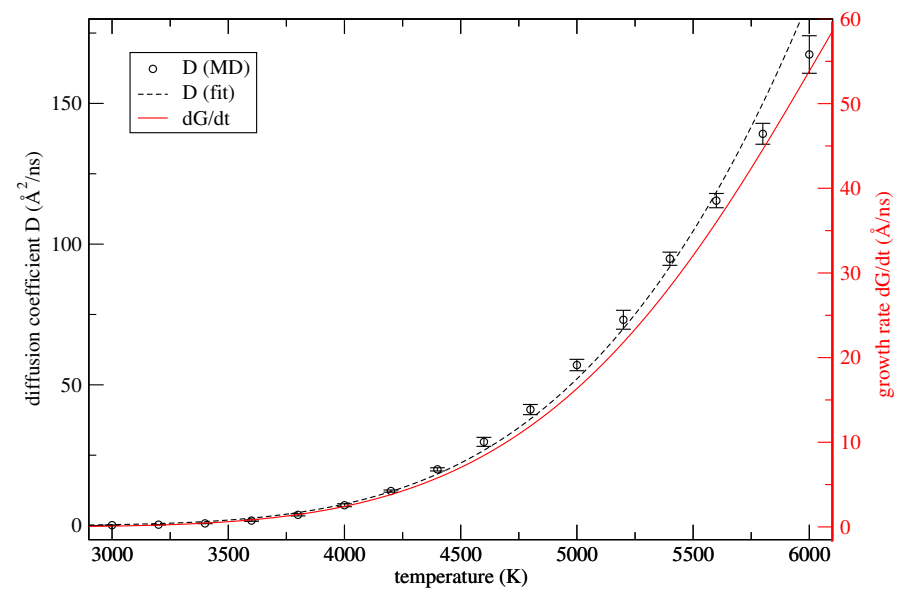

Figure 4: Profiles of the diffusion coefficient for $\mathrm{MoS}_{2}$ within the amorphous region and of the linear growth rate of the crystal nuclei (see Eq. 5) as functions of the temperature.

with diffusion when $T \ll T_{m}\left(\dot{G} \sim \frac{D}{a}\right)$, and slows down to 0 as $T$ approaches $T_{m}$. Once an explicit expression for the nucleation and growth rates has been worked out, they can then be used in order to calculate the fraction $Y$ of material which has been transformed into the stable phase as a function of time (Avrami equation $\left.{ }^{65}\right):{ }^{4}$

$$
-\ln (1-Y)=\frac{\pi}{3} h \dot{N} \dot{G}^{2} t^{3}
$$

\footnotetext{
${ }^{4}$ Please note that if spherical particles are considered, a similar equation is derived ${ }^{64}$ with the notable difference that the time entries raised at the fourth power.
} 
In Fig. 5 a double logarithmic plot for the same MD trajectory reported in Fig. 2 is shown. As can be seen, there is an initiation period during which a few critical nuclei have to be

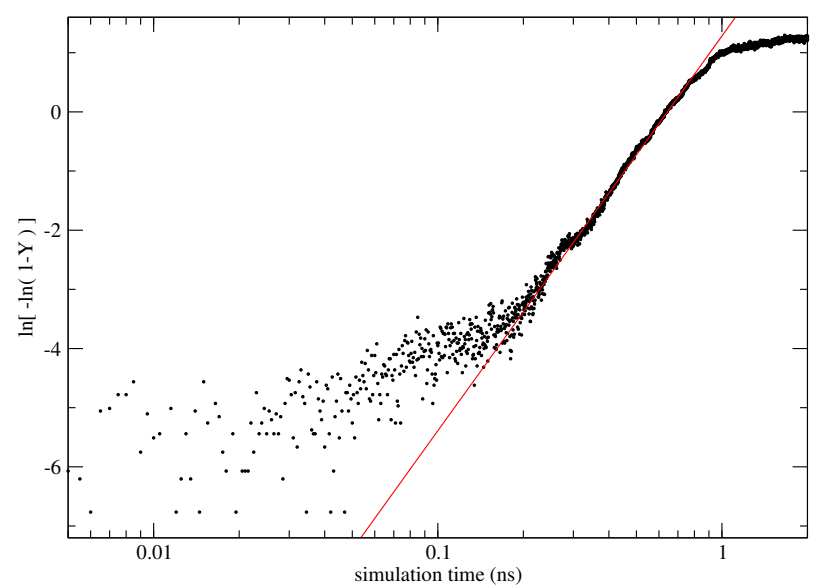

Figure 5: Plot of $\ln [-\ln (1-Y)]$ versus time (logarithmic scale) for the trajectory with load equal to $50 \mathrm{GPa}$, temperature equal to $4000 \mathrm{~K}$ and density equal to $2.09 \mathrm{~g} \mathrm{~cm}^{-3}$. The red straight line is the linear fit obtained employing Eq. 6.

formed before the transformation process can start. The Avrami equation cannot account for this initial transient period, since it assumes a constant nucleation rate that is not the proper description for the process at the nanoscale ${ }^{5}$. At any rate, as soon as the nucleation process starts to take place, $\ln [-\ln (1-Y)]$ follows the linear behavior predicted by the Avrami equation quite well. It is important to note that the slope of the linear fit is very close to three. This implies that the mathematical law governing this process is exactly in the form of Eq. 6 (i.e., the negative logarithm of the untransformed fraction grows with the third power of the time). As shown in the Supporting Information - Section 6, this is directly related to the fact that the growth of the crystal nuclei happens mainly in two dimensions, thus confirming the appropriateness of considering cylindrical particles instead of spherical ones. At longer times, the deviation from linearity can be easily explained by considering that both the nucleation and the growth processes are stopped by the unavailability of untransformed material, thus leading to a steady value.

\footnotetext{
${ }^{5}$ Given the energy barrier for nucleation $\Delta G^{*}$, a stochastic model (e.g., kinetic Monte Carlo) can be worked out giving a statistically accurate description of the process, although this goes beyond the scope of this paper. For our goals, it suffices to disregard the initial transient from the linear fit.
} 
It is also possible to define a characteristic time $\tau$ as the time at which the system comprises an equal amount of metastable and crystal phase. It follows that

$$
\tau=\left(\frac{3 \ln 2}{\pi h \dot{N} \dot{G}^{2}}\right)^{\frac{1}{3}}
$$

The values of $\tau$ at different temperatures can be calculated directly from MD simulations, whilst $\dot{G}$ can be evaluated through Eq. 5. Therefore, by plugging Eq. 4 into Eq. 6, one can obtain the unknown parameters $(A$ and $\sigma)$ through a fitting procedure. The results of such a fit are reported in Fig. 6 (the actual values of $A$ and $\sigma$ are $21892 \AA^{-3} \mathrm{~ns}^{-1}$ and $2.49 \mathrm{kcal}$ $\mathrm{mol}^{-1} \AA^{-2}$ ). Evidently, the equations coming from CNT (i.e., Eqs. 4, 5 and 6) fit reasonably

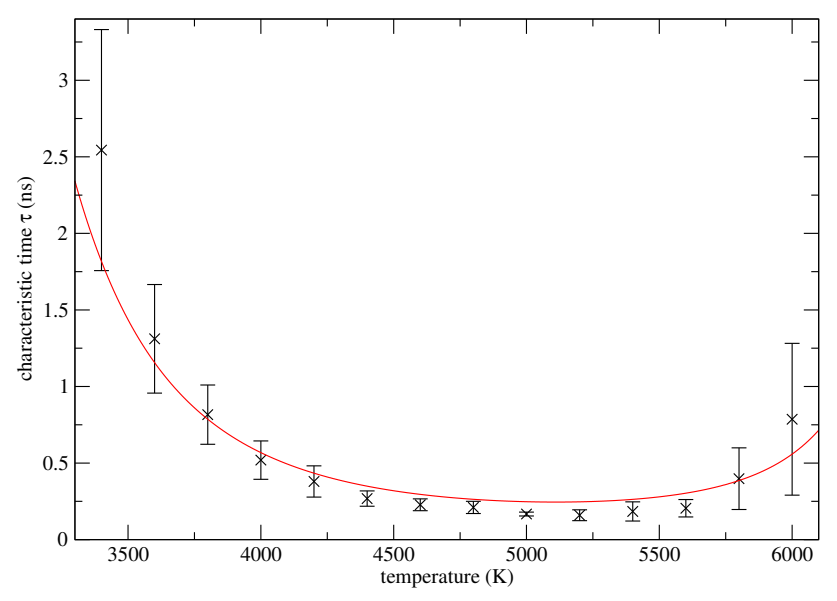

Figure 6: Plot of the characteristic time of crystal formation $\tau$ (see Eqs. 4, 5 and 7) as a function of temperature. The " $\mathrm{x}$ " symbols represent the values calculated from the MD trajectories (error bars are the standard deviations obtained from the different coating densities) whilst the red line represents the result of the fitting procedure.

well the MD data and they are able to reproduce the observed characteristic time trends. In fact, it is possible to derive a temperature for which the crystallization process proceeds at maximum speed (approx. $5110 \mathrm{~K}$ ) and for both higher and lower temperature values $\tau$ shows a monotonic increase. Finally, since all terms entering in Eqs. 4, 5 and 6 are known, it is possible to extrapolate $\tau$ for temperature values outside the explored window. The results of such an extrapolation are reported in Tab. 1. At room temperature, the characteristic times for conversion from the amorphous to the crystal structure are extremely high, meaning 
Table 1: Results of the extrapolation procedure by using Eqs. 3, 4, 5 and 7. In the last column, the letters "m", "h", "d" and "y" stand for minutes, hours, days and years respectively

\begin{tabular}{ccccc}
$\begin{array}{c}\mathrm{T} \\
(\mathrm{K})\end{array}$ & $\begin{array}{c}\Delta G^{*} \\
\left(\mathrm{kcal} \mathrm{mol}^{-1}\right)\end{array}$ & $\begin{array}{c}\dot{N} \\
\left(\AA^{-3} \mathrm{~ns}^{-1}\right)\end{array}$ & $\begin{array}{c}\dot{G} \\
\left(\AA \mathrm{ns}^{-1}\right)\end{array}$ & $\tau$ \\
\hline 2500 & 69.0 & $2.0 \cdot 10^{-2}$ & $7.7 \cdot 10^{-3}$ & $45 \mathrm{~ns}$ \\
2000 & 62.8 & $3.0 \cdot 10^{-3}$ & $1.7 \cdot 10^{-4}$ & $1.1 \mu \mathrm{s}$ \\
1500 & 57.7 & $8.6 \cdot 10^{-5}$ & $2.8 \cdot 10^{-7}$ & $0.25 \mathrm{~ms}$ \\
1000 & 53.3 & $4.9 \cdot 10^{-8}$ & $7.8 \cdot 10^{-13}$ & $15 \mathrm{~s}$ \\
900 & 52.5 & $3.9 \cdot 10^{-9}$ & $1.1 \cdot 10^{-14}$ & $11 \mathrm{~m}$ \\
800 & 51.8 & $1.6 \cdot 10^{-10}$ & $5.4 \cdot 10^{-17}$ & $17 \mathrm{~h}$ \\
700 & 51.0 & $2.6 \cdot 10^{-12}$ & $5.7 \cdot 10^{-20}$ & $270 \mathrm{~d}$ \\
600 & 50.3 & $1.1 \cdot 10^{-14}$ & $6.2 \cdot 10^{-24}$ & $\sim 10^{3} \mathrm{y}$ \\
500 & 49.6 & $4.8 \cdot 10^{-18}$ & $1.7 \cdot 10^{-29}$ & $\sim 10^{8} \mathrm{y}$ \\
400 & 48.9 & $4.3 \cdot 10^{-23}$ & $8.2 \cdot 10^{-38}$ & $\sim 10^{15} \mathrm{y}$ \\
300 & 48.2 & $1.7 \cdot 10^{-31}$ & $1.1 \cdot 10^{-51}$ & $\sim 10^{27} \mathrm{y}$
\end{tabular}

that at $300 \mathrm{~K}$ it is almost impossible to observe the transformation process. This prediction agrees with the experimental observations, since coatings made of amorphous molybdenum disulfide are known to be stable over at least a period of months. One can notice that as the temperature decreases, the metastable phase is further destabilized and consequently the nucleation barrier $\Delta G^{*}$ decreases as well. Nevertheless, the available thermal energy diminishes more quickly and, consequently, the nucleation rate decreases by almost 30 orders of magnitude as the temperature goes down from 2500 to $300 \mathrm{~K}$. The growth rate decrease is even more drastic (almost 50 orders of magnitude), as a consequence of the reduction of the diffusivity in the amorphous materials at room temperature. On the other hand, from the values in Tab. 1 one can notice that for having a characteristic time in the order of seconds or minutes, the temperature needs to be raised to $900-1000 \mathrm{~K}$. This range is compatible with the temperatures that can be reached at the contact during a tribological measurements ${ }^{66-68}$ or in experiments where samples are irradiated with laser beams ${ }^{40,44,45,69}$ and therefore, under the assumptions made within the present treatment, our results can provide a framework for the interpretation of the experimental findings.

Finally, we investigated the effect of the sliding speed on the crystal formation process. To do this, we performed other sets of MD simulations obtained by imposing a shearing velocity, 
namely 1 and $5 \mathrm{~m} \mathrm{~s}^{-1}$, at different temperatures (in the range between 3000 and $5000 \mathrm{~K}$ ) for all initial densities of the amorphous phase, whilst keeping fixed the duration of the MD trajectory $(2 \mathrm{~ns})$. The profiles with the physical quantities of interest for typical trajectories are reported in Fig. 7 (all other profiles can be found in the Supporting Information - Section 8). The trends shown in the panels on the right are qualitatively identical to those reported
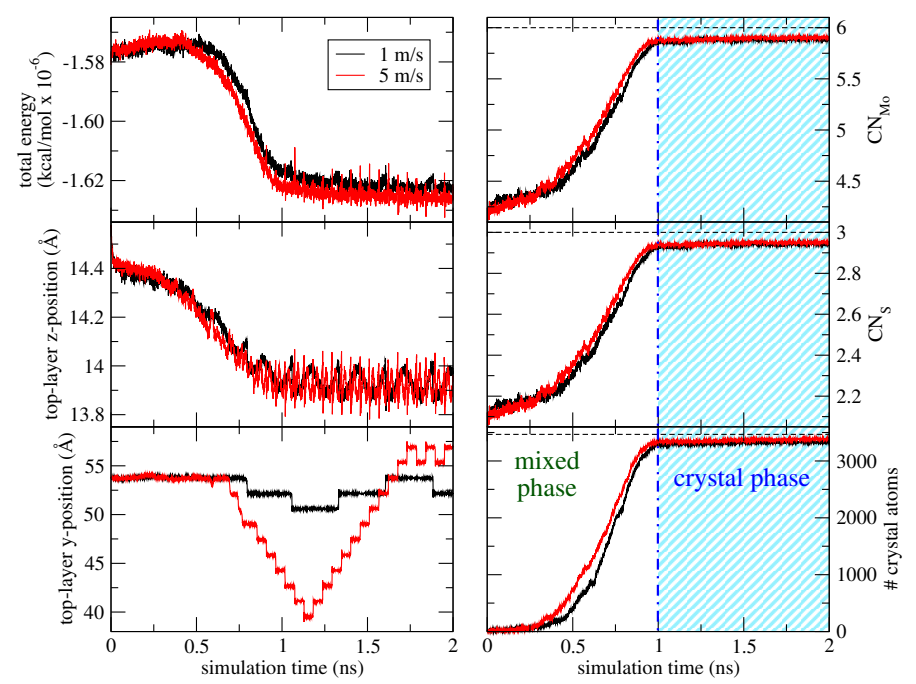

Figure 7: Plot reporting the same physical quantities as in Fig. 2 (except for the bottom left panel, which is a plot of the position in the $y$ direction of the topmost rigid layer), here for the cases of imposing a sliding speed. All MD trajectories are calculated with load equal to $50 \mathrm{GPa}$, temperature equal to $4000 \mathrm{~K}$ and density equal to $2.09 \mathrm{~g} \mathrm{~cm}^{-3}$.

in Fig. 2 (i.e., without sliding) and confirm the observation of the transformation from the amorphous to crystal structure. On the other hand, in the panels on the left, one can see that the formation of regular patterns can be observed during the sliding dynamics. In particular, for both the value of the total system energy and the position in the $z$-direction of the top layer, periodic (with a frequency proportional to the sliding speed) variations can be observed after the system has transformed from amorphous to crystalline. These changes become even more evident if we look at the profile of the position in the $y$-direction of the top layer, where it is possible to distinguish between two different regimes: a first part where the motion is smooth, and a second one (after the formation of the crystalline layer), where the dynamics becomes discontinuous, showing sudden "jumps" from a stable 
configuration to another one. These are characteristic traits of the so-called "stick-slip" dynamics which is known to happen during the sliding of crystalline layered materials. ${ }^{70}$ Let us focus now on the kinetics of the crystal formation. Results regarding the characteristic times $\tau$ at different temperatures and with an imposed sliding speed are reported in Fig. 8, together with the results from the previous set of simulations. The points corresponding to

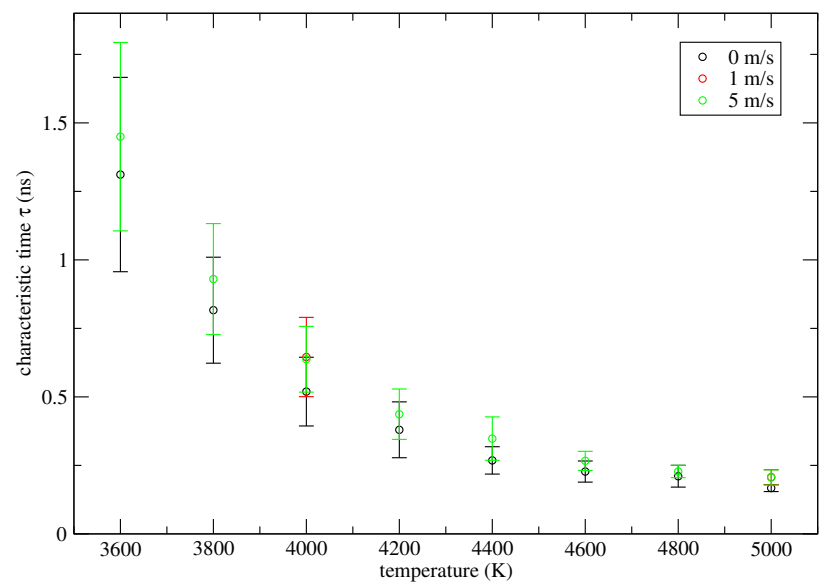

Figure 8: Same plot as in Fig. 6, here also showing the cases of imposing a sliding speed.

the sliding conditions fall very close (within the uncertainty limits) to those coming from the simulations where no shearing speed has been imposed, therefore we can conclude that the effect of the sliding speed on the crystal formation process under these extreme conditions is negligible. Since the fundamental mechanisms behind the crystal formation may be the same in both (sliding and no-sliding) conditions, on the basis of the results reported in Fig. 8, we hypothesize that, in real-world experiments the dominant role of the sliding velocity is to provide energy to the surfaces in contact (in the form of heat) necessary for activating the nucleation and growth processes that lead to the formation of a crystal layer.

\section{Concluding remarks}

In summary, we have studied, by non-equilibrium molecular dynamics simulations, the formation process of crystalline $\mathrm{MoS}_{2}$ from amorphous structures confined between rigid substrates. We have systematically investigated the effect of temperature, initial sample density, 
and sliding speed of the confining substrates, and have found the ideal operating conditions necessary to achieve the crystal formation. We have shown that our results can be explained in the framework of the classical nucleation theory. In particular, we have elucidated the microscopic mechanisms of crystal formation, characterized by initial formation of nuclei followed by their growth, and we have shown that the process is mainly ruled by system diffusivity, while amorphous density and sliding speed play a minor role. Therefore, we conclude that the absence of crystal formation at temperatures below $3500 \mathrm{~K}$ is due to the low diffusivity suppressing the growth of nuclei in the limited time window accessible to our simulations.

We are aware that our simulations represent an idealized system confined between perfectly rigid, crystalline surfaces that certainly contribute to the emergence of nucleation. Nevertheless, our results can provide a reasonable theoretical framework to explain the experimental findings on the crystallization of $\mathrm{MoS}_{2}$ due to sliding or exposure to laser or electron beams. Supported by our results, we suggest that such crystallization processes can be explained by heat transfer and the consequent increase of temperature at the sliding interface or on the surface exposed to laser/electron beam. Our simulation identifies the energy threshold required to trigger the amorphous-to-crystalline transformation, which can be used to identify optimum sliding conditions or processing parameters to produce crystalline $\mathrm{MoS}_{2}$.

\section{Acknowledgement}

The authors acknowledge the use of the HPC clusters administered by IT4Innovations National Supercomputing Center, University of Ostrava, Czech Republic and the Abel Cluster, owned by the University of Oslo and the Norwegian metacenter for High Performance Com-

puting (NOTUR), and operated by the Department for Research Computing at USIT, the University of Oslo IT-department, in the completion of this work. This publication was sup- 
ported by the Czech Science Foundation, project 16-11516Y. This work was partly supported by COST Action MP1303.

\section{Supporting Information Available}

The file "si.pdf" is available free of charge. It includes information about:

- the estimation of the melting temperature and enthalpy for the force field for $\mathrm{MoS}_{2}$ employed in this study under load and confinement conditions

- the estimation of structural parameters from equilibrium MD simulations

- the algorithm for the identification of the crystal network

- time profiles of physical quantities for MD trajectories without sliding speed and temperatures between 3000 and $6000 \mathrm{~K}$ for all coating densities

- MD snapshots taken from the trajectory reported in the main text at different times (temperature $4000 \mathrm{~K}$ and initial density of the amorphous $2.09 \mathrm{~g} \mathrm{~cm}^{-3}$ )

- the derivation of formulas used in the main text about nucleation and growth of cylindrical particles

- the estimation of the diffusion coefficient for $\mathrm{MoS}_{2}$ in the amorphous phase from MD simulations

- time profiles of physical quantities for MD trajectories with sliding speeds equal to 1 and $5 \mathrm{~m} \mathrm{~s}^{-1}$ and temperatures between 3000 and $6000 \mathrm{~K}$ for all coating densities

This material is available free of charge via the Internet at http://pubs.acs.org/.

\section{References}

(1) Lansdown, A. R. Molybdenum Disulfide Lubrication; Elsevier, 1999. 
(2) Schönfeld, B.; Huang, J. J.; Moss, S. C. Anisotropic Mean-Square Displacements (MSD) in Single Crystals of 2H- and 3R-MoS 2 . Acta Crystallographica Section B 1983, B39, 404-407.

(3) Jaramillo, T. F.; Jørgensen, K. P.; Bonde, J.; Nielsen, J. H.; Horch, S.; Chorkendorff, I. Identification of Active Edge Sites for Electrochemical $\mathrm{H}_{2}$ Evolution from $\mathrm{MoS}_{2}$ Nanocatalysts. Science 2007, 317, 100-102.

(4) Chianelli, R. R.; Siadati, M. H.; la Rosa, M. P. D.; Berhault, G.; Wilcoxon, J. P.; Jr., R. B.; Abrams, B. L. Catalytic Properties of Single Layers of Transition Metal Sulfide Catalytic Materials. Catalysis Reviews: Science and Engineering 2006, 48, 141.

(5) Zaman, S.; Smith, K. J. A Review of Molybdenum Catalysts for Synthesis Gas Conversion to Alcohols: Catalysts, Mechanisms and Kinetics. Catalysis Reviews: Science and Engineering 2012, 54, 41-132.

(6) Li, Y.; Li, Y.-L.; Araujo, C. M.; Luo, W.; Ahuja, R. Single-layer $\mathrm{MoS}_{2}$ as an efficient photocatalyst. Catalysis Science \& Technology 2013, 3, 2214-2220.

(7) Late, D. J.; Huang, Y.; Liu, B.; Acharya, J.; Shirodkar, S. N.; Luo, J.; Yan, A.; Charles, D.; Waghmare, U. V.; Dravid, V. P.; Rao, C. N. R. Sensing Behavior of Atomically Thin-Layered $\mathrm{MoS}_{2}$ Transistors. ACS Nano 2013, 7, 4879-4891.

(8) Sarkar, D.; Liu, W.; Xie, X.; Anselmo, A. C.; Mitragotri, S.; Banerjee, K. MoS 2 FieldEffect Transistor for Next-Generation Label-Free Biosensors. ACS Nano 2014, 8, 39924003.

(9) Yang, X.; Li, J.; Liang, T.; Ma, C.; Zhang, Y.; Chen, H.; Hanagata, N.; Su, H.; $\mathrm{Xu}, \mathrm{M}$. Antibacterial activity of two-dimensional $\mathrm{MoS}_{2}$ sheets. Nanoscale 2014, 6, $10126-10133$. 
(10) Yin, W.; Yan, L.; Yu, J.; Tian, G.; Zhou, L.; Zheng, X.; Zhang, X.; Yong, Y.; Li, J.; Gu, Z.; Zhao, Y. High-Throughput Synthesis of Single- Layer $\mathrm{MoS}_{2}$ Nanosheets as a Near- Infrared Photothermal-Triggered Drug Delivery for Effective Cancer Therapy. ACS Nano 2014, 8, 6922-6933.

(11) Radisavljevic, B.; Radenovic, A.; Brivio, J.; Giacometti, V.; Kis, A. Single-layer $\mathrm{MoS}_{2}$ transistors. Nature Nanotechnology 2011, 6, 147-150.

(12) Tsai, M.-L.; Su, S.-H.; Chang, J.-K.; Tsai, D.-S.; Chen, C.-H.; Wu, C.-I.; Li, L.-J.; Chen, L.-J.; He, J.-H. Monolayer $\mathrm{MoS}_{2}$ Heterojunction Solar Cells. ACS Nano 2014, 8, 8317-8322.

(13) Clauss, F. J. Solid Lubricants and Self-Lubricating Solids; Academic Press, 1972.

(14) Song, I.; Park, C.; Choi, H. C. Synthesis and properties of molybdenum disulphide: from bulk to atomic layers. RSC Advances 2015, 5, 7495-7514.

(15) Moser, J.; Liao, H.; Lévy, F. Texture characterisation of sputtered $\mathrm{MoS}_{2}$ thin films by cross-sectional TEM analysis. Journal of Physics D: Applied Physics 1990, 23, 624-626.

(16) Hilton, M. R.; Fleischauer, P. D. TEM lattice imaging of the nanostructure of earlygrowth sputter-deposited $\mathrm{MoS}_{2}$ solid lubricant films. Journal of Materials Research 1990, 5, 406-421.

(17) Moser, J.; Lévy, F. Growth mechanisms and near-interface structure in relation to orientation of $\mathrm{MoS}_{2}$ sputtered thin films. Journal of Materials Research 1992, 7, 734740.

(18) Donnet, C.; Mogne, T. L.; Martin, J. M. Superlow friction of oxygen-free $\mathrm{MoS}_{2}$ coatings in ultrahigh vacuum. Surface 83 Coatings Technology 1993, 62, 406-411.

(19) Lévy, F.; Moser, J. High-resolution cross-sectional studies and properties of molybdenite coatings. Surface 83 Coatings Technology 1994, 68/69, 433-438. 
(20) Moser, J.; Lévy, F. Random stacking in $\mathrm{MoS}_{2-x}$ sputtered thin films. Thin Solid Films 1994, $240,56-59$.

(21) Spalvins, T. Tribological Properties of Sputtered $\mathrm{MoS}_{2}$ Films in Relation to Film Morphology. Thin Solid Films 1980, 73, 291-297.

(22) Mikkelsen, N. J.; Chevallier, J.; Sørensen, G.; Straede, C. A. Friction and wear measurements of sputtered $\mathrm{MoS}_{x}$ films amorphized by ion bombardment. Applied Physics Letters 1988, 52, 1130-1132.

(23) Hilton, M. R.; Bauer, R.; Fleischauer, P. D. Tribological Performance and Deformation of Sputter-Deposited $\mathrm{MoS}_{2}$ Solid Lubricant Films During Sliding Wear and Indentation Contact. Thin Solid Films 1990, 188, 219-236.

(24) Fleischauer, P. D.; Hilton, M. R.; Bauer, R. Effects of microstructure and adhesion on performance of sputter-deposited $\mathrm{MoS}_{2}$ solid lubricant coatings. Mechanics of Coatings. 1990; pp 121-128.

(25) Alam, T.; Wang, B.; Pulavarthy, R.; Haque, M. A.; Muratore, C.; Glavin, N.; Roy, A. K.; Voevodin, A. A. Domain engineering of physical vapor deposited twodimensional materials. Applied Physics Letters 2014, 105, 213110.

(26) Martin, J. M.; Donnet, C.; Mogne, T. L.; Epicier, T. Superlubricity of molybdenum disulfide. Physical Review B 1993, 48, 10583-10586.

(27) Lince, J. R.; Fleischauer, P. D. Crystallinity of rf-sputtered MoS $\mathrm{M}_{2}$ films. Journal of Materials Research 1987, 2, 827-838.

(28) Moser, J.; Lévy, F. MoS $2_{x}$ lubricating films: structure and wear mechanisms investigated by cross-sectional transmission electron microscopy. Thin Solid Films 1993, 228, $257-260$. 
(29) Martin, J. M.; Pascal, H.; Donnet, C.; Mogne, T. L.; Loubet, J. L.; Epicier, T. Superlubricity of $\mathrm{MoS}_{2}$ : crystal orientation mechanisms. Surface $\&$ Coatings Technology 1994, 68/69, 427-432.

(30) Seitzman, L. E.; Bolster, R. N.; Singer, I. L.; Wegand, J. C. Relationship of Endurance to Microstructure of IBAD MoS 2 Coatings. Tribology Transactions 1995, 38, 445-451.

(31) Gustavsson, F.; Jacobson, S. Diverse mechanisms of friction induced self-organisation into a low-friction material - An overview of $\mathrm{WS}_{2}$ tribofilm formation. Tribology International 2016, 101, 340-347.

(32) Domínguez-Meister, S.; Conte, M.; Igartua, A.; Rojas, T. C.; Sánchez-López, J. C. Self-Lubricity of $\mathrm{WSe}_{x}$ Nanocomposite Coatings. ACS Applied Materials $\mathcal{E}$ Interfaces 2015, 7, 7979-7986.

(33) Kim, H. I.; Lince, J. R. Direct visualization of sliding-induced tribofilm on $\mathrm{Au} / \mathrm{MoS}_{2}$ nanocomposite coatings by c-AFM. Tribology Letters 2007, 26, 61-65.

(34) Scharf, T. W.; Goeke, R. S.; Kotula, P. G.; Prasad, S. V. Synthesis of Au-MoS 2 Nanocomposites: Thermal and Friction- Induced Changes to the Structure. ACS Applied Matererials $\& 3$ Interfaces 2013, 5, 11762-11767.

(35) Wahl, K. J.; Seitzman, L. E.; Bolster, R. N.; Singer, I. L. Low-friction, high-endurance, ion-beam-deposited Pb-Mo-S coatings. Surface $\mathscr{6}$ Coatings Technology 1995, 73, 152159.

(36) Polcar, T.; Cavaleiro, A. Review on self-lubricant transition metal dichalcogenide nanocomposite coatings alloyed with carbon. Surface \&3 Coatings Technology 2011, 206, 686-695.

(37) Gustavsson, F.; Jacobson, S.; Cavaleiro, A.; Polcar, T. Ultra-low friction W-S-N solid lubricant coating. Surface \& Coatings Technology 2013, 232, 541-548. 
(38) Ghosh, S. K.; Srivastava, C.; Nath, S.; Celis, J. P. Simple Formation of Nanostructured Molybdenum Disulfide Thin Films by Electrodeposition. International Journal of Electrochemistry 2013, 2013, 138419.

(39) Berdinsky, A. S.; Chadderton, L. T.; Yoo, J. B.; Gutakovsky, A. K.; Fedorov, V. E.; Mazalov, L. N.; Fink, D. Structural changes of $\mathrm{MoS}_{2}$ nano-powder in dependence on the annealing temperature. Applied Physics A 2005, 80, 61-67.

(40) Fei, L.; Lei, S.; Zhang, W.-B.; Lu, W.; Lin, Z.; Lam, C. H.; Chai, Y.; Wang, Y. Direct TEM observations of growth mechanisms of two-dimensional $\mathrm{MoS}_{2}$ flakes. Nature Communications 2016, 7, 12206.

(41) Jin, Z.; Shin, S.; Kwon, D. H.; Han, S.-J.; Min, Y.-S. Novel chemical route for atomic layer deposition of $\mathrm{MoS}_{2}$ thin film on $\mathrm{SiO}_{2} / \mathrm{Si}$ substrate. Nanoscale 2014, 6, 1445314458.

(42) Zink, N.; Therese, H. A.; Pansiot, J.; Yella, A.; Banhart, F.; Tremel, W. In Situ Heating TEM Study of Onion-like $\mathrm{WS}_{2}$ and $\mathrm{MoS}_{2}$ Nanostructures Obtained via MOCVD. Chemistry of Materials 2008, 20, 65-71.

(43) Hanzawa, A.; Shimada, T.; Hasegawa, T.; Sato, T.; Kamino, T.; Yonezawa, T. In-Situ TEM Observations of the Crystallization Process of Solution-Prepared $\mathrm{MoS}_{2}$ Amorphous Particles. Journal of Nanoscience and Nanotechnology 2009, 9, 6736-6740.

(44) Jagminas, A.; Niaura, G.; Žalnèravičius, R.; Trusovas, R.; Račiukaitis, G.; Jasulaitiene, V. Laser Light Induced Transformation of Molybdenum Disulphide-Based Nanoplatelet Arrays. Scientific Reports 2016, 6, 37514.

(45) Nguyen, D. N.; Nguyen, L. N.; Nguyen, P. D.; Thu, T. V.; Nguyen, A. D.; Tran, P. D. Crystallization of Amorphous Molybdenum Sulfide Induced by Electron or Laser Beam and Its Effect on $\mathrm{H}_{2}$-Evolving Activities. Journal of Physical Chemistry C 2016, 120, $28789-28794$. 
(46) Zabinski, J. S.; Donley, M. S.; John, P. J.; Dyhouse, V. J.; Safriet, A. J.; McDevitt, N. T. Crystallization of Molybdenum Disulfide Films Deposited by Pulsed Laser Ablation. MRS Proceedings 1990, 201, 195-200.

(47) Hromadová, L.; Martoňák, R.; Tosatti, E. Structure change, layer sliding, and metallization in high-pressure $\mathrm{MoS}_{2}$. Physical Review B 2013, 87, 144105.

(48) Curry, J. F.; Argibay, N.; Babuska, T.; Nation, B.; Martini, A.; Strandwitz, N. C.; Dugger, M. T.; Krick, B. A. Highly Oriented $\mathrm{MoS}_{2}$ Coatings: Tribology and Environmental Stability. Tribology Letters 2016, 64, 11.

(49) Rasmussen, F. A.; Thygesen, K. S. Computational 2D Materials Database: Electronic Structure of Transition-Metal Dichalcogenides and Oxides. Journal of Physical Chemistry C 2015, 119, 13169-13183.

(50) Schweiger, H.; Raybaud, P.; Kresse, G.; Toulhoat, H. Shape and Edge Sites Modifications of $\mathrm{MoS}_{2}$ Catalytic Nanoparticles Induced by Working Conditions: A Theoretical Study. Journal of Catalysis 2002, 207, 76-87.

(51) Curry, J. F.; Wilson, M. A.; Luftman, H. S.; Strandwitz, N. C.; Argibay, N.; Chandross, M.; Sidebottom, M. A.; Krick, B. A. Impact of Microstructure on $\mathrm{MoS}_{2}$ Oxidation and Friction. ACS Applied Materials 83 Interfaces 2017, 9, 28019-28026.

(52) Morita, Y.; Onodera, T.; Suzuki, A.; Sahnoun, R.; Koyama, M.; Tsuboi, H.; Hatakeyama, N.; Endou, A.; Takaba, H.; Kubo, M.; Carpio, C. A. D.; Shin-yoshi, T.; Nishino, N.; Suzuki, A.; Miyamoto, A. Development of a new molecular dynamics method for tribochemical reaction and its application to formation dynamics of $\mathrm{MoS}_{2}$ tribofilm. Applied Surface Science 2008, 254, 7618-7621.

(53) Plimpton, S. Fast Parallel Algorithms for Short-Range Molecular Dynamics. Journal of Computational Physics 1995, 117, 1-19. 
(54) Liang, T.; Phillpot, S. R.; Sinnott, S. B. Parametrization of a reactive many-body potential for Mo-S systems. Physical Review B 2009, 79, 245110.

(55) Liang, T.; Phillpot, S. R.; Sinnott, S. B. Erratum: Parametrization of a reactive manybody potential for Mo-S systems [Phys. Rev. B 79, 245110 (2009)]. Physical Review B 2012, 85, 199903(E).

(56) Nicolini, P.; Polcar, T. A comparison of empirical potentials for sliding simulations of $\mathrm{MoS}_{2}$. Computational Materials Science 2016, 115, 158-169.

(57) Varshney, V.; Patnaik, S. S.; Muratore, C.; Roy, A. K.; Voevodin, A. A.; Farmer, B. L. MD simulations of molybdenum disulphide $\left(\mathrm{MoS}_{2}\right)$ : Force-field parameterization and thermal transport behavior. Computational Materials Science 2010, 48, 101-108.

(58) Nosé, S. A unified formulation of the constant temperature molecular dynamics methods. Journal of Chemical Physics 1984, 81, 511-519.

(59) Hoover, W. G. Canonical dynamics: Equilibrium phase-space distributions. Physical Review A 1985, 31, 1695-1697.

(60) Yoon, C. S.; Megusar, J. Molecular Dynamic Simulation of Amorphous Carbon and Graphite Interface. Interface Science 1995, 3, 85-100.

(61) Parrinello, M.; Rahman, A. Polymorphic transitions in single crystals: A new molecular dynamics method. Journal of Applied Physics 1981, 52, 7182-7190.

(62) Schneider, T.; Stoll, E. Molecular-dynamics study of a three-dimensional onecomponent model for distortive phase transitions. Physical Review B 1978, 17, 13021322.

(63) Doltsinis, N. L.; Sprik, M. Theoretical $\mathrm{pK}_{a}$ estimates for solvated $\mathrm{P}(\mathrm{OH})_{5}$ from coordination constrained CarâĂŞParrinello molecular dynamics. Physical Chemistry Chemical Physics 2003, 5, 2612-2618. 
(64) Debenedetti, P. G. Metastable Liquids; Princeton University Press, 1997.

(65) Avrami, M. Kinetics of Phase Change. I General Theory. Journal of Chemical Physics 1939, $\%, 1103$.

(66) Karmandonov, A. F.; Brudnyi, A. I.; Kiryanov, A. M. Influence of Degree of Oxidation of Molybdenum Disulfide on its Lubricating Capacity. Chemistry and Technology of Fuels and Oils 1974, 10, 798-800.

(67) Kragelsky, I. V.; Dobychin, M. N.; Kombalov, V. S. Friction and Wear - Calculation Methods; Pergamon Press, 1981.

(68) Bowden, F. P.; Ridler, K. E. W. Physical properties of surfaces - III - The surface temperature of sliding metals - The temperature of lubricated surfaces. Proceedings of the Royal Society of London A: Mathematical, Physical and Engineering Sciences 1936, 154, 640-656.

(69) Moore, D. S.; McGrane, S. D. Raman temperature measurement. Journal of Physics: Conference Series 2014, 500, 192011.

(70) Liu, X.-Z.; Ye, Z.; Dong, Y.; Egberts, P.; Carpick, R. W.; Martini, A. Dynamics of Atomic Stick-Slip Friction Examined with Atomic Force Microscopy and Atomistic Simulations at Overlapping Speeds. Physical Review Letters 2015, 114, 146102. 
Graphical TOC Entry

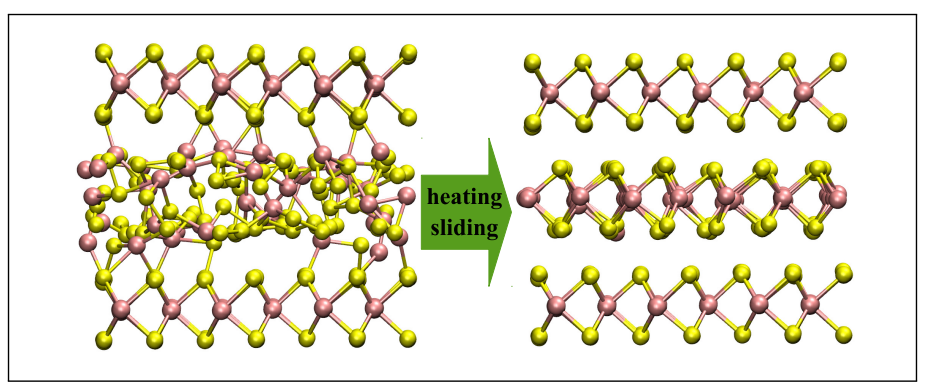

